\title{
Criteria for identifying meals from continuous recordings of the behaviour of grazing dairy cows
}

\author{
AJ Rook, CA Huckle \\ Institute of Grassland and Environmental Research, North Wyke, Okehampton, Devon EX20 2SB, UK
}

\begin{abstract}
Animals eat in discrete meals separated by inter-meal intervals. Short intra-meal intervals also occur. In order to define a meal, a criterion is required by which these two types of interval may be distinguished. This is usually achieved by examining the frequency distribution of intervals of different duration. A series of piecewise linear regressions of frequency on interval duration is fitted, with successively greater break points. The break point of the best fitting regression is taken as the meal criterion with all intervals shorter than the breakpoint regarded as intra-meal intervals. Other methods involving explicit assumptions about the underlying distributions of intra- and inter-meal intervals have also been proposed.
\end{abstract}

In this study, the meal criterion for HolsteinFriesian cows, grazing a perennial ryegrasswhite clover sward, without supplementation, was examined. Eating, ruminating or idling activity, to a resolution of 1 minute, was obtained using a system which automatically recorded jaw movements. Thirty eight 24-hour recordings were used in this analysis.

Piecewise linear regression analysis indicated a meal criterion of 4-5 minutes. However, examination of the frequency distribution revealed a second break point at an interval of approximately 20 minutes. In order to understand this occurrence further studies were conducted.

Recordings for each of the 7 cows represented in the dataset were analyzed separately. All animals showed a meal criterion of 3-4 minutes and all showed some evidence of a second break point which could not therefore be attributed to between animal differences.

Intervals composed of ruminating or idling alone or both together (mixed) were analyzed separately. The frequency of short intervals was greatest for ruminating and lowest for mixed intervals. A clear 4-5 minute meal criterion was again evident in all cases. The secondary break point also appeared in each case. It is not therefore due to the different frequency of different types of interval.

Periods between am and pm milkings and pm and am milkings were analyzed separately to ascertain if meal criteria changed over the day. There was no evidence that this was the case.

Once a meal criterion has been determined data may be smoothed to remove both intrameal intervals and very small meals. In this study smoothing was achieved by examining a window of 9 consecutive minutes and assigning to the midpoint minute the activity (eating, ruminating or idling) which occurred most often during this period. Examination of the intra-meal intervals eliminated in this way suggested that two types of meal were present in the smoothed data. The majority of meals were characterised by long sustained periods of eating with small intra-meal intervals, that typically accounted for $0.4-0.6 \%$ of the total meal. However, some meals were much more broken with intra-meal intervals accounting for up to $40 \%$ of the total time. This suggests there may be some merit in a system which characterises the nature of each meal as well as simply identifying it. Intra-meal intervals appeared to occur in a random manner and not, for example, to be concentrated at the end of the meal.

Part of the increased frequency of intervals between 5 and 20 minutes that caused the second breakpoint to occur appeared to be due to the breaking up of long intervals by periods of 1 or 2 minutes eating. These very short 'meals' contribute very little to total intake. It is therefore suggested that rather than obtaining meal criteria from raw data, the data should be successively smoothed, removing meals and intervals of 1,2 etc. minutes. Piecewise linear models may then be fitted to this smoothed data. 